\title{
IDENTIFICATION OF RESISTORS IN ELECTRICAL NETWORKS
}

\author{
Soon-Yeong Chung
}

\begin{abstract}
The purpose of this work is to identify the internal structure of the electrical networks with data obtained from only a part of network or the boundary of network. To be precise, it is discussed whether we can identify resistors or electrical conductivities of each link inside networks by the measurement of voltage on the boundary which is induced by a prescribed current on the boundary. As a result, it is shown that the structure of the resistor network can be determined uniquely by only one pair of the data (current, voltage) on the boundary, if the resistors satisfy an appropriate condition. Besides, several useful results about the energy functionals, which means the electrical power, are included.
\end{abstract}

\section{Introduction}

The problem of discovering the detailed inner structure of the electrical network from a collection of boundary measurements can be seen as a type of inverse problem, analogous to those arising in tomography. For example, problems of interest include checking connectivity, performance, reliability of the networks and so on. In particular, when we have some problem on a part of the network or when we are in need of finding such part having problems, it is almost impossible to investigate the whole network, since the network may be too vast and its structure or connectivity be too complicated. For this reason, the study of the inverse problem to recover the whole network with partial data is becoming increasingly important for practical applications.

In the graph theory, problems involving graph identification with a partial information have been among the most important and famous open problems ([6]). Most of the work on this subject has concentrated on spectral graph theory (see [4], [8], [12], [9], [11], [10], [13], [14] and [16]). Thus far, spectral graph theory has been one of the most significant tools used studying graphs, and it has led to noteworthy progress in the study along these questions. But,

Received February 20, 2009; Revised August 22, 2009.

2000 Mathematics Subject Classification. Primary 94C15; Secondary 94C05, 94C12.

Key words and phrases. resistor network, Thompson's principle, inverse problem.

The author acknowledges the support from grants KRF-2003-041-C00023. 
as it is well known, graphs are not in general completely characterized by their spectra (see [13], p. 66).

The purpose of this work is to see what data we need, in order to identify the internal structure of the electrical resistor networks. To be precise, it is discussed whether we can identify resistors or electrical conductivities of each link inside networks by the measurement of voltage on the boundary which is induced by a prescribed current on the boundary. In particular, we are interested in finding such data that depends on only a minimum number of measurements. As a result of this study, it is shown that the structure of the resistor network can be determined uniquely by only one pair of the data (current, voltage) on the boundary, if the resistors satisfy an appropriate condition.

The following global uniqueness result is one of the main result of this paper, which is stated in the final section:

Theorem. Let $\left(\bar{S}, R_{1}\right)$ and $\left(\bar{S}, R_{2}\right)$ be resistor networks with the same boundary $\partial S$ and let $J_{1}$ and $J_{2}$ be Kirchhoff's flows on $\left(\bar{S}, R_{1} ; \partial S\right)$ and $\left(\bar{S}, R_{2} ; \partial S\right)$, respectively, which are commonly induced by a voltage function $\sigma$ on $\partial S$.

Assume the followings:

(i) $J_{1}(z)=J_{2}(z), z \in \partial S$,

(ii) $R_{1} \leq R_{2}$ (i.e., $R_{1}(x, y) \leq R_{2}(x, y), x, y \in \bar{S}$ with $\left.x \sim y\right)$. Then

(i) $J_{1}(x, y)=J_{2}(x, y), x, y \in \bar{S}$,

(ii) $R_{1}(x, y)=R_{2}(x, y)$ whenever $J_{1}(x, y) \neq 0$ or $J_{2}(x, y) \neq 0$.

The second conclusion $R_{1}(x, y)=R_{2}(x, y)$ above is exactly what we want to have. In fact, it shows not only whether each pair of nodes is connected by a link, but also how nice the link is. Thus the author believes that there are possible applications of the above result in fault-testing of IC circuits or VLSI design to see whether it is normal or not.

In fact, the monotonicity condition $R_{1} \leq R_{2}$ was borrowed from the continuous case. Some form of monotonicity has been considered in the inverse conductivity problem of continuous body (refer to [1], [2], [7], [17], [18], [19] and so on).

We organized this paper as follows: In Section 1, we give several mathematical characterization of electrical currents and prove a unique existence of current with a prescribed values on the boundary in a resistor network. Section 2 is devoted to discuss the energy of resistor networks and Thompson's principle. In Section 3, we prove the unique determinateness of resistors by only one-time measurement of the current and voltage on the boundary. To do this we introduce the Dirichlet principle, which can be understood as the converse of Thompson's principle. 


\section{Flows and potential functions}

We begin with some definitions and notions frequently used throughout this paper.

By a resistor network $G:=(V, R)$ we mean mathematically a pair consisting of finite sets $V$ of nodes (or vertices) and a function $R: V \times V \rightarrow[0, \infty]$ with $R(x, x)=0, x \in V$, called the resistor. When $R(x, y) \neq \infty$, the nodes $x$ and $y$ are said to be adjacent and denoted by $x \sim y$.

A network $G$ is said to be connected if for every pair of nodes $x$ and $y$ there exist a sequence (termed a path) of vertices $x=x_{0}, x_{1}, x_{2}, \ldots, x_{n-1}, x_{n}=y$ such that $x_{j-1} \sim x_{j}$ for $j=1,2, \ldots, n$. Throughout this paper, all the networks in our concern are assumed to be connected. In addition, we agree with $0 \cdot \infty=0$ and $\frac{1}{\infty}=0$.

The degree $d_{R} x$ of a node $x$ in a resistor network $G$ with a resistor $R$ is defined by

$$
d_{R} x:=\sum_{y \in \bar{S}}[R(x, y)]^{-1} .
$$

Definition 1.1. For a network $G=(V, R)$ with a subset $S$ of $V$, a function $j: V \times V \rightarrow \mathbb{R}$ is said to be a flow on $(V, R ; \partial S)$ if it satisfies the followings:

(i) $j(x, y)=-j(y, x), x, y \in V$,

(ii) $j(x, y)=0$ if $x \nsim y$ or $x=y$,

(iii) $j(x):=\sum_{y \in V} j(x, y)=0, x \in S$,

where $\partial S$ denotes the set satisfying $V=S \cup \partial S$.

When a flow $j$ on $(V, R ; \partial S)$ is given, the set $S$ and $\partial S$ is said to be the interior and the boundary of $V$, respectively. In what follows, when we consider a network with an interior $S$ and a boundary $\partial S$, the set of its nodes is denoted by $\bar{S}$, instead of $V$.

Every flow necessarily has the following property on networks, which is a well known fact:

Lemma 1.2. Every flow $j$ on $(\bar{S}, R ; \partial S)$ satisfies $\sum_{z \in \partial S} j(z)=0$.

Proof. By the definition of a flow, we have

$$
\begin{aligned}
\sum_{z \in \partial S} j(z) & =\sum_{z \in \partial S} j(z)+\sum_{x \in S} j(x) \\
& =\sum_{x \in \bar{S}} j(x) \\
& =\sum_{x \in \bar{S}} \sum_{y \in \bar{S}} j(x, y) \\
& =\frac{1}{2} \sum_{x \in \bar{S}} \sum_{y \in \bar{S}}[j(x, y)+j(y, x)]=0 .
\end{aligned}
$$


Definition 1.3. A function $f: \bar{S} \rightarrow \mathbb{R}$ is said to be a potential function of a flow $j$ on $(\bar{S}, R ; \partial S)$ if

$$
f(x)-f(y)=j(x, y) R(x, y)
$$

for every pair of nodes $x, y$ in $\bar{S}$ with $x \sim y$.

If $f$ is a potential function of a flow $j$ on $(\bar{S}, R ; \partial S)$, then (iii) in the definition above implies

$$
\Delta_{R} f(x):=\sum_{y \in \bar{S}}[f(x)-f(y)][R(x, y)]^{-1}=0, \quad x \in S .
$$

Here, $\Delta_{R}$ has been known as the combinatorial Laplacian (see [3], [8], [12], and so on).

Remark 1.4. In general, a flow $j$ may not have its potential function. Consider a resistor network with all resistors $1 \mathrm{ohm}$ and boundary $\partial S=\{0,5\}$

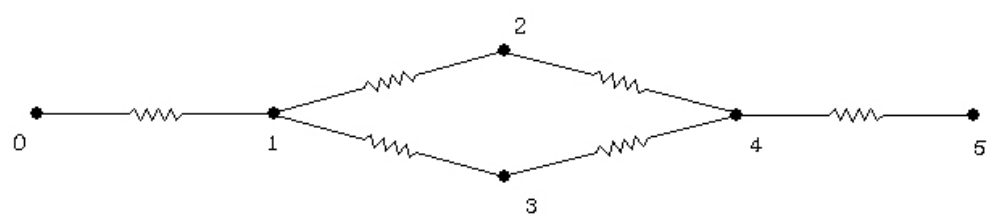

and a flow $j$ defined by

$$
\begin{aligned}
& j(0,1)=1, \\
& j(1,2)=j(2,4)=\alpha, \\
& j(1,3)=j(3,4)=\beta, \\
& j(4,5)=1,
\end{aligned}
$$

where $\alpha+\beta=1$. Suppose that $j$ has a potential function $f$. Then

$$
\begin{aligned}
& f(0)-f(1)=j(0,1)=1, \\
& f(1)-f(2)=j(1,2)=\alpha, \\
& f(2)-f(4)=j(2,4)=\alpha, \\
& f(1)-f(3)=j(1,3)=\beta, \\
& f(3)-f(4)=j(3,4)=\beta .
\end{aligned}
$$

The the second and third formula give

$$
f(4)=f(1)-2 \alpha
$$

and the last two formula give

$$
f(4)=f(1)-2 \beta .
$$

This leads a contradiction whenever $\alpha \neq \beta$.

The following is a necessary and sufficient condition for a flow to have a potential function:

Theorem 1.5. For a flow $j$ on $(\bar{S}, R ; \partial S)$, the followings are equivalent: 
(i) $j$ has a potential function.

(ii) $\sum_{j=0}^{N} j\left(x_{i}, x_{i+1}\right) R\left(x_{i}, x_{i+1}\right)=0$ for vertices along every closed path $x_{0} \sim$ $x_{1} \sim x_{2} \sim \cdots \sim x_{N} \sim x_{N+1}=x_{0}$.

(iii) For every pair of nodes $x$ and $y$,

$$
\sum_{j=0}^{M} j\left(x_{i}, x_{i+1}\right) R\left(x_{i}, x_{i+1}\right)=\sum_{i=1}^{N} j\left(y_{i}, y_{i+1}\right) R\left(y_{i}, y_{i+1}\right)
$$

for vertices along paths from $x$ to $y$

$$
\begin{aligned}
& x=x_{0} \sim x_{1} \sim \cdots \sim x_{M} \sim x_{M+1}=y, \\
& x=y_{0} \sim y_{1} \sim \cdots \sim y_{N} \sim y_{N+1}=y .
\end{aligned}
$$

Proof. The equivalence of (ii) and (iii) is easy. Suppose that (i) is true. Then we have a (potential) function $f$ such that

$$
f(x)-f(y)=j(x, y) R(x, y)
$$

for every $x$ and $y$ in $\bar{S}$ with $x \sim y$. Then

$$
\begin{aligned}
& \sum_{j=0}^{N} j\left(x_{i}, x_{i+1}\right) R\left(x_{i}, x_{i+1}\right) \\
= & \sum_{j=0}^{N}\left[f\left(x_{i}\right)-f\left(x_{j}\right)\right] \\
= & \left\{f\left(x_{0}\right)-f\left(x_{1}\right)\right\}+\left\{f\left(x_{1}\right)-f\left(x_{2}\right)\right\}+\cdots+\left\{f\left(x_{N}\right)-f\left(x_{N+1}\right)\right\} \\
= & 0
\end{aligned}
$$

since $x_{0}=x_{N+1}$. Now we assume that (iii) is true. Let $x_{0} \in S$ be fixed and $x \in \bar{S}$ be arbitrary. Consider a path

$$
x_{0} \sim x_{1} \sim x_{2} \sim \cdots \sim x_{N} \sim x_{N+1}=x .
$$

Define

$$
f(x)=f\left(x_{0}\right)+\sum_{i=0}^{N} j\left(x_{i+1}, x_{i}\right) R\left(x_{i+1}, x_{i}\right) .
$$

Then (iii) implies that $f$ is well defined being independent of the choice of paths. Then for arbitrary $x$ and $y$ in $S$ with $x \sim y$ we take paths from $x_{0}$ to $x$ and from $x_{0}$ to $y$, respectively, as follows:

$$
x_{0} \sim x_{1} \sim x_{2} \sim \cdots \sim x_{N}=x
$$

and

$$
x_{0} \sim x_{1} \sim x_{2} \sim \cdots \sim x_{N}=x \sim y
$$

Then

$$
f(y)=f\left(x_{0}\right)+\sum_{i=0}^{N-1} j\left(x_{i+1}, x_{i}\right) R\left(x_{x+1}, x_{i}\right)+j(y, x) R(y, x)
$$




$$
=f(x)+j(y, x) R(y, x),
$$

which implies that $f$ is a potential function of the flow $j$.

Remark 1.6. (i) The condition (ii) above can be understood nothing but Kirchhoff's voltage law: the sum of the voltage difference along any closed path is 0 .

(ii) Once we have a potential function of a flow, then it is true that the flow has a unique potential function up to an additional constant. To see this, let $f$ and $g$ be potential functions of a flow $j$. Then

$$
f(x)-f(y)=j(x, y) R(x, y)=g(x)-g(y)
$$

and so that

$$
f(x)-g(x)=f(y)-g(y)
$$

for every pair of nodes $x, y$ in $\bar{S}$ with $x \sim y$. Then the connectedness of the network shows that $f-g$ is constant.

Definition 1.7. A flow $j$ on $(\bar{S}, R, \partial S)$ is called Kirchhoff's flow if it has a potential function.

Remark 1.8. In what follows, we reserve $J$ for Kirchhoff's flow and $j$ for general ones.

We note that Theorem 1.5 gives a characterization of Kirchhoff's flows. Another equivalent characterization of the Kirchhoff's flow will be given later in Section 3.

Now we follow the work of Fan Chung and her collaborators in [8], [12] and [11] to discuss the combinatorial Laplacian $\Delta_{R}$ on a network $\bar{S}$ defined on (1.1). Since it is easy to see that $\Delta_{R}$ is a linear operator on a finite dimensional vector space, it can be considered as a matrix. In this viewpoint, one can easily check that $\Delta_{R}$ is a symmetric matrix indexed by nodes in $\bar{S}$ as follows:

$$
\Delta_{R}(x, y)=\left\{\begin{array}{cl}
d_{R} x, & \text { if } x=y \\
-\frac{1}{R(x, y)}, & \text { otherwise. }
\end{array}\right.
$$

Consider a function $f$ on $\bar{S}$ as a $N$-dimensional vector, where $N$ denotes the number of nodes in $\bar{S}$. Then we use Rayleigh's principle to get the first eigenvalue

$$
\begin{aligned}
\lambda_{0} & =\min _{f \neq 0} \frac{\left\langle f, \Delta_{R} f\right\rangle}{\langle f, f\rangle} \\
& =\min _{f \neq 0} \frac{1}{2} \frac{\sum_{x, y \in \bar{S}}[f(x)-f(y)]^{2}[R(x, y)]^{-1}}{\sum_{y \in \bar{S}} f(x)^{2}} \\
& =0,
\end{aligned}
$$

by taking $f$ as a nonzero constant function for which the minimum occurs. Here, we used the notation $\langle f, g\rangle:=\sum_{x \in \bar{S}} f(x) g(x)$. The same argument can 
be applied to see that the next eigenvalue

$$
\lambda_{1}=\min _{\sum f(x)=0, f \neq 0} \frac{\left\langle f, \Delta_{R} f\right\rangle}{\langle f, f\rangle}
$$

is positive. Therefore, we see that $\Delta_{R}$ is a nonnegative definite symmetric matrix having the eigenvalues

$$
\lambda_{0}=0<\lambda_{1} \leq \lambda_{2} \leq \cdots \leq \lambda_{N-1},
$$

and corresponding eigenfunctions

$$
\Phi_{0}, \Phi_{1}, \Phi_{2}, \ldots, \Phi_{N-1},
$$

which are orthonormal in the sense that for each pair of distinct $i$ and $j$

$$
\sum_{x \in \bar{S}} \Phi_{i}(x) \cdot \Phi_{j}(x)=0,
$$

while, for all $j$,

$$
\sum_{x \in \bar{S}}\left|\Phi_{j}(x)\right|^{2}=1
$$

Here, we note that $\Phi_{0}(x)=\frac{1}{\sqrt{N}}$ for all $x \in \bar{S}$.

Now we are ready to prove the existence theorem of Kirchhoff's flow with the prescribed current.

Theorem 1.9. Let $(\bar{S}, R ; \partial S)$ be a resistor network and $\gamma$ be any function defined on $\partial S$ with $\sum_{z \in \partial S} \gamma(z)=0$. Then there exists a unique Kirchhoff's flow $J$ such that

$$
J(z)=\gamma(z), \quad z \in \partial S
$$

Proof. First, we are going to find a function satisfying a couple of equations

$$
\sum_{y \in \bar{S}}[f(x)-f(y)][R(x, y)]^{-1}=0, \quad x \in S
$$

and

$$
\sum_{y \in \bar{S}}[f(z)-f(y)][R(z, y)]^{-1}=0, \quad z \in \partial S .
$$

These equations can be expressed equivalently as

$$
\Delta_{R} f(x)=g(x), \quad x \in \bar{S},
$$

where

Now consider the orthogonal expansion

$$
g(x)=\left\{\begin{array}{l}
0, \quad x \in S \\
\gamma(x), \quad x \in \partial S
\end{array}\right.
$$

$$
f(x)=\sum_{j=0}^{N-1} a_{j} \Phi_{j}(x), \quad x \in \bar{S},
$$


where $a_{j}=\left\langle f, \Phi_{j}\right\rangle, j=0,1,2, \ldots, N-1$. Then it follows that

$$
\begin{aligned}
\lambda_{j} \cdot a_{j} & =\left\langle f, \Delta_{R} \Phi_{j}\right\rangle \\
& =\left\langle\Delta_{R} f, \Phi_{j}\right\rangle \\
& =\left\langle g, \Phi_{j}\right\rangle,
\end{aligned}
$$

so that we have

$$
a_{j}=\frac{1}{\lambda_{j}}\left\langle g, \Phi_{j}\right\rangle, \quad j=1,2, \ldots, N-1 .
$$

On the other hand, $a_{0}$ is an arbitrary constant, since

$$
\begin{aligned}
\lambda_{0} \cdot a_{0} & =\left\langle g, \Phi_{0}\right\rangle \\
& =\frac{1}{\sqrt{N}} \sum_{z \in \partial S} \gamma(z) \\
& =0 .
\end{aligned}
$$

Hence, it turns out that

$$
f(x)=\frac{a_{0}}{\sqrt{N}}+\sum_{j=1}^{N-1} \frac{1}{\lambda_{j}} \sum_{z \in \partial S} \gamma(z) \Phi_{j}(z) \Phi_{j}(x), x \in \bar{S} .
$$

Conversely, a simple computation shows that $f$ in (1.4) satisfies the equation (1.3).

Then a flow $J$ defined by $J(x, y)=[f(x)-f(y)] / R(x, y)$ gives a Kirchhoff's flow such that $J(z)=\gamma(z), z \in \partial S$.

To prove the uniqueness, let $J_{1}$ and $J_{2}$ be Kirchhoff's flows with potential functions $f_{1}$ and $f_{2}$, respectively, such that $J_{1}(z)=\gamma(z)=J_{2}(z), z \in \partial S$. Then the functions $f_{1}$ and $f_{2}$ satisfy the equation (1.3) so that they should be written in the form as in (1.4), only with different constants $a_{0}$. This implies that $f_{1}=f_{2}+$ (constant) on $\bar{S}$. Then it follows that for every $x$ and $y$ in $\bar{S}$,

$$
\begin{aligned}
J_{1}(x, y) & =\left[f_{1}(x)-f_{1}(y)\right] / R(x, y) \\
& =\left[f_{2}(x)-f_{2}(y)\right] / R(x, y) \\
& =J_{2}(x, y)
\end{aligned}
$$

which completes the proof.

\section{Energies of flows}

For a flow $j$ on a resistor network $(\bar{S}, R ; \partial S)$ and a function $f$ on $\bar{S}$, the energy $E_{R}(j ; f)$ of the flow $j$ with respect to the function $f$ is defined by

$$
E_{R}(j ; f)=\frac{1}{2} \sum_{x, y \in \bar{S}} j(x, y)[f(x)-f(y)] .
$$


In particular, if the flow $j$ has a potential function $f$, then the energy $E_{R}(j ; f)$ can be written by

$$
E_{R}(j ; f)=\frac{1}{2} \sum_{x, y \in \bar{S}}[j(x, y)]^{2} R(x, y) .
$$

In this case, we use the notation $E_{R}(j)$ instead of $E_{R}(j ; f)$ for simplicity.

Theorem 2.1 (Energy Conservation). For a flow $j$ on $(\bar{S}, R ; \partial S)$ and a function $f$ on $\bar{S}$, we have

$$
E_{R}(j ; f)=\sum_{z \in \partial S} f(z) j(z) .
$$

Proof. Since $j(x, y)=-j(y, x)$ and $j(x)=0$ for $x, y \in S$, it follows that

$$
\begin{aligned}
& \sum_{x, y \in \bar{S}} j(x, y)[f(x)-f(y)] \\
= & \sum_{x \in \bar{S}} f(x)\left[\sum_{y \in \bar{S}} j(x, y)\right]-\sum_{y \in \bar{S}} f(y)\left[\sum_{x \in \bar{S}} j(x, y)\right] \\
= & \sum_{x \in \bar{S}} f(x) j(x)-\sum_{y \in \bar{S}} f(y)[-j(y)] \\
= & 2 \sum_{z \in \bar{S}} f(z) j(z) \\
= & 2 \sum_{z \in \partial S} f(z) j(z)
\end{aligned}
$$

which completes the proof.

The following is a well known fact to engineers (see [15]).

Theorem 2.2 (Thompson's Principle). Let $(\bar{S}, R ; \partial S)$ be a resistor network and $\gamma: \partial S \rightarrow \mathbb{R}$ be a function with $\sum_{z \in \partial S} \gamma(z)=0$. If $J$ is a Kirchhoff's flow on $(\bar{S}, R ; \partial S)$ with $\left.J\right|_{\partial S}=\gamma$, then

$$
E_{R}(J)=\min _{j \in A} \frac{1}{2} \sum_{x, y \in \bar{S}}[j(x, y)]^{2} R(x, y),
$$

where $A$ is the set of all flow $j$ on $(\bar{S}, R ; \partial S)$ with $\left.j\right|_{\partial S}=\gamma$.

Proof. Let $f$ be a potential function of $J$. For $j \in A$, we define $i(x, y)=$ $j(x, y)-J(x, y)$ for all $x, y \in \bar{S}$. Then $i$ defines a flow on $\bar{S}$ and satisfies

$$
\begin{aligned}
i(z) & =\sum_{y \in \bar{S}} i(z, y) \\
& =\sum_{y \in \bar{S}} j(z, y)-\sum_{y \in \bar{S}} J(z, y)
\end{aligned}
$$




$$
\begin{aligned}
& =j(z)-J(z) \\
& =0
\end{aligned}
$$

for all $z \in \partial S$. Then

$$
\begin{aligned}
\sum_{x, y \in \bar{S}}[j(x, y)]^{2} R(x, y)= & \sum_{x, y \in \bar{S}}[i(x, y)+J(x, y)]^{2} R(x, y) \\
= & \sum_{x, y \in \bar{S}}[i(x, y)]^{2} R(x, y)+\sum_{x, y \in \bar{S}}[J(x, y)]^{2} R(x, y) \\
& +2 \sum_{x, y \in \bar{S}} i(x, y) J(x, y) R(x, y) .
\end{aligned}
$$

Now using the fact

$$
J(x, y) R(x, y)=f(x)-f(y)
$$

and Theorem 2.1 we obtain

$$
\begin{aligned}
& \sum_{x, y \in \bar{S}}[j(x, y)]^{2} R(x, y) \\
= & \sum_{x, y \in \bar{S}}[i(x, y)]^{2} R(x, y)+\sum_{x, y \in \bar{S}}[J(x, y)]^{2} R(x, y)+\sum_{z \in \partial S} f(z) i(z) \\
= & \sum_{x, y \in \bar{S}}[i(x, y)]^{2} R(x, y)+\sum_{x, y \in \bar{S}}[J(x, y)]^{2} R(x, y) \\
\geq & \sum_{x, y \in \bar{S}}[J(x, y)]^{2} R(x, y),
\end{aligned}
$$

since $i(z)=0, z \in \partial S$. This completes the proof.

For a given network $(\bar{S}, R ; \partial S)$, let $\gamma: \partial S \rightarrow \mathbb{R}$ be a function with

$$
\sum_{z \in \partial S} \gamma(z)=0
$$

Then by Theorem 1.9 there exists a unique Kirchhoff's flow $j_{R}$ such that $j_{R}(z)=\gamma(z), z \in \partial S$. Then we define

$$
F(R):=E_{R}\left(j_{R}\right)=\frac{1}{2} \sum_{x, y \in \bar{S}}\left[j_{R}(x, y)\right]^{2} R(x, y) .
$$

Theorem 2.3 (Concavity on Resistors). Let $\gamma: \partial S \rightarrow \mathbb{R}$ be a fixed function with $\sum_{z \in \partial S} \gamma(z)=0$. For resistor networks $\left(\bar{S}, R_{1} ; \partial S\right)$ and $\left(\bar{S}, R_{2} ; \partial S\right)$, we have followings:

(i) $F\left(R_{1}+R_{2}\right) \geq F\left(R_{1}\right)+F\left(R_{2}\right)$.

(ii) $F\left(R_{1}\right) \leq F\left(R_{2}\right)$ whenever $R_{1} \leq R_{2}$ (i.e., $R_{1}(x, y) \leq R_{2}(x, y), x, y \in \bar{S}$ with $x \sim y)$.

(iii) $F(r R)=r F(R)$ for every $r>0$.

(iv) $F\left(\frac{R_{1}+R_{2}}{2}\right) \geq \frac{F\left(R_{1}\right)+F\left(R_{2}\right)}{2}$. 
Proof. We use Thompson's principle to get

$$
\begin{aligned}
& F\left(R_{1}+R_{2}\right) \\
= & \frac{1}{2} \sum_{x, y \in \bar{S}}\left[j_{\left(R_{1}+R_{2}\right)}(x, y)\right]^{2}\left[R_{1}(x, y)+R_{2}(x, y)\right] \\
= & \frac{1}{2} \sum_{x, y \in \bar{S}}\left[j_{\left(R_{1}+R_{2}\right)}(x, y)\right]^{2} R_{1}(x, y)+\frac{1}{2} \sum_{x, y \in \bar{S}}\left[j_{\left(R_{1}+R_{2}\right)}(x, y)\right]^{2} R_{2}(x, y) \\
\geq & \frac{1}{2} \sum_{x, y \in \bar{S}}\left[j_{R_{1}}(x, y)\right]^{2} R_{1}(x, y)+\frac{1}{2} \sum_{x, y \in \bar{S}}\left[j_{R_{2}}(x, y)\right]^{2} R_{2}(x, y) \\
= & F\left(R_{1}\right)+F\left(R_{2}\right),
\end{aligned}
$$

which gives (i). Now we prove (ii).

$$
\begin{aligned}
F\left(R_{2}\right) & =\frac{1}{2} \sum_{x, y \in \bar{S}}\left[j_{R_{2}}(x, y)\right]^{2} R_{2}(x, y) \\
& \geq \frac{1}{2} \sum_{x, y \in \bar{S}}\left[j_{R_{2}}(x, y)\right]^{2} R_{1}(x, y) \\
& \geq \frac{1}{2} \sum_{x, y \in \bar{S}}\left[j_{R_{1}}(x, y)\right]^{2} R_{1}(x, y) \\
& =F\left(R_{1}\right),
\end{aligned}
$$

where the last inequality follows also from Thompson's principle.

Now let $j_{R}$ be a Kirchhoff's flow such that $j_{R}(z)=\gamma(z), z \in \partial S$ and $f_{R}$ be its potential function on a resistor network $(S, R, \partial S)$. Then it follows that

$$
f_{R}(x)-f_{R}(y)=j_{R}(x, y) R(x, y), x \sim y
$$

and in turn

$$
r f_{R}(x)-r f_{R}(y)=j_{R}(x, y) \cdot r R(x, y), x \sim y,
$$

which implies that $j_{R}$ is still a Kirchhoff's flow of the network $(S, r R, \partial S)$. Then it turns out that

$$
\begin{aligned}
F(r R) & =\frac{1}{2} \sum_{x, y \in \bar{S}}\left[j_{r R}(x, y)\right]^{2} r R(x, y) \\
& =r \cdot \frac{1}{2} \sum_{x, y \in \bar{S}}\left[j_{R}(x, y)\right]^{2} R(x, y) \\
& =r F(R),
\end{aligned}
$$

which gives (iii). Then (iv) is easily obtained from (i) and (iii). 


\section{Inverse problems}

Now we prove the converse of Thompson's principle that is essential for us to derive the main theorem. This result seems to be not only interesting itself but also very useful in the inverse problem. In fact, such a theorem has been known as the Dirichlet principle in the theory of nonlinear PDE's.

Theorem 3.1 (Dirichlet Principle). Let $(\bar{S}, R ; \partial S)$ be a resistor network and $\gamma: \partial S \rightarrow \mathbb{R}$ be a function with $\sum_{z \in \partial S} \gamma(z)=0$. If $J \in A$ minimizes $\frac{1}{2} \sum_{x, y \in \bar{S}}[j(x, y)]^{2} R(x, y)$ on $A$, then $J$ is a unique Kirchhoff's flow on $(\bar{S}, R ; \partial S)$, where $A$ is the set of all flow $j$ on $(\bar{S}, R ; \partial S)$ with $\left.j\right|_{\partial S}=\gamma$.

Proof. The uniqueness is easy from the condition $\left.J\right|_{\partial S}=\gamma$ and Theorem 1.9. Now let $k$ be a flow on $\bar{S}$ such that $k(z)=0, z \in \partial S$. Define a function $\alpha$ on $\mathbb{R}$ by

$$
\alpha(\tau)=E(J+\tau k), \quad \tau \in \mathbb{R} .
$$

Then $\alpha$ is clearly a differentiable function with respect to $\tau$ and

$$
\begin{aligned}
\frac{d \alpha}{d \tau}= & \frac{1}{2} \sum_{x, y \in \bar{S}}[J(x, y)]^{2} R(x, y)+\tau \sum_{x, y \in \bar{S}} J(x, y) k(x, y) R(x, y) \\
& +\frac{\tau^{2}}{2} \sum_{x, y \in \bar{S}}[k(x, y)]^{2} R(x, y) .
\end{aligned}
$$

Since $J+\tau k \in A$ and $J$ is a minimizer, it follows that $\frac{d \alpha}{d \tau}(0)=0$, i.e.,

$$
\sum_{x, y \in \bar{S}} J(x, y) k(x, y) R(x, y)=0 .
$$

Now applying Theorem 1.9 we can find a Kirchhoff's flow $J_{0}$ and its potential function $h$ such that $\left.J_{0}\right|_{\partial S}=\gamma$ and

$$
h(x)-h(y)=J_{0}(x, y) R(x, y), x \sim y .
$$

Then it follows from Theorem 2.1 (Energy Conservation) that

$$
\begin{aligned}
& \frac{1}{2} \sum_{x, y \in \bar{S}}\left[J_{0}(x, y)\right]^{2} R(x, y) \\
= & \frac{1}{2} \sum_{x, y \in \bar{S}} J_{0}(x, y)[h(x)-h(y)] \\
= & \sum_{z \in \partial S} h(z) \gamma(z) \\
= & \frac{1}{2} \sum_{x, y \in \bar{S}} J(x, y)[h(x)-h(y)] \\
= & \frac{1}{2} \sum_{x, y \in \bar{S}} J(x, y) J_{0}(x, y) R(x, y)
\end{aligned}
$$




$$
\begin{aligned}
& =\frac{1}{2} \sum_{x, y \in \bar{S}} J(x, y)\left[J_{0}(x, y)-J(x, y)\right] R(x, y)+\frac{1}{2} \sum_{x, y \in \bar{S}}[J(x, y)]^{2} R(x, y) \\
& =\frac{1}{2} \sum_{x, y \in \bar{S}}[J(x, y)]^{2} R(x, y)
\end{aligned}
$$

where the last equality follows from (3.1). On the other hand, in view of (3.1) we see that

$$
\sum_{x, y \in \bar{S}} J(x, y)\left[J(x, y)-J_{0}(x, y)\right] R(x, y)=0 .
$$

In other words,

$$
\sum_{x, y \in \bar{S}}[J(x, y)]^{2} R(x, y)=\sum_{x, y \in \bar{S}} J(x, y) J_{0}(x, y) R(x, y) .
$$

Then it follows from (3.2) and (3.3) that

$$
\begin{aligned}
& \frac{1}{2} \sum_{x, y \in \bar{S}}\left[J(x, y)-J_{0}(x, y)\right]^{2} R(x, y) \\
= & \frac{1}{2} \sum_{x, y \in \bar{S}}[J(x, y)]^{2} R(x, y)+\frac{1}{2} \sum_{x, y \in \bar{S}}\left[J_{0}(x, y)\right]^{2} R(x, y) \\
& -\sum_{x, y \in \bar{S}} J(x, y) J_{0}(x, y) R(x, y) \\
= & 0,
\end{aligned}
$$

which implies that $J(x, y)=J_{0}(x, y), x, y \in \bar{S}$. Therefore, $J$ is a Kirchhoff's flow on $(\bar{S}, R ; \partial S)$.

Combining the arguments in the above proof, we actually obtain the following:

Corollary 3.2. For a flow $J$ on a resistor network $(\bar{S}, R ; \partial S)$ with $\left.J\right|_{\partial S}=\gamma$, the followings are equivalent:

(i) $J$ is a Kirchhoff's flow on $(\bar{S}, R ; \partial S)$.

(ii) $J$ minimizes $\frac{1}{2} \sum_{x, y \in \bar{S}}[j(x, y)]^{2} R(x, y)$ on $A$, where $A$ is the set of all flow $j$ on $(\bar{S}, R, \partial S)$ with $\left.j\right|_{\partial S}=\gamma$.

(iii) $\begin{aligned} \sum_{x, y \in \bar{S}} J(x, y) k(x, y) R(x, y)=0 \text { for every flow } k \text { on }(\bar{S}, R, \partial S) \text { with } \\ \left.k\right|_{\partial S=0}\end{aligned}$

Example. Consider a resistor network 




with all resistors $1 \mathrm{ohm}$. Here, $\{0,6\}$ is designated as boundary. Then every flow $j$ with $j(0)=1$ and $j(6)=-1$, can be written as follows:

$$
\begin{aligned}
& j(0,1)=1, \\
& j(5,6)=1, \\
& j(1,2)=j(2,5)=a, \\
& j(1,3)=j(3,5)=b, \\
& j(1,4)=j(4,5)=c,
\end{aligned}
$$

where $a, b$ and $c$ are arbitrary numbers such that $a+b+c=1$. Then

$$
E(j)=1^{2}+2 a^{2}+2 b^{2}+2 c^{2}+(-1)^{2}
$$

and $E(j)$ assumes a minimum when $a=b=c=1 / 3$. The potential function of the minimizing flow is given by

$$
\begin{gathered}
f(0)=d, \quad f(1)=d-1 \\
f(2)=f(3)=f(4)=d-\frac{4}{3} \\
f(5)=d-\frac{5}{3}, \quad f(6)=d-\frac{\tau}{3} .
\end{gathered}
$$

Here, $d$ is an arbitrary number.

Now we are in a position to state and prove the main theorem of this paper.

Theorem 3.3 (Uniqueness). Let $\left(\bar{S}, R_{1}\right)$ and $\left(S, R_{2}\right)$ be resistor networks with the same boundary $\partial S$ and let $J_{1}$ and $J_{2}$ be Kirchhoff's flows on $\left(\bar{S}, R_{1} ; \partial S\right)$ and $\left(\bar{S}, R_{2} ; \partial S\right)$, respectively, which are commonly induced by a voltage function $\sigma$ on $\partial S$.

Assume the followings:

(i) $J_{1}(z)=J_{2}(z), z \in \partial S$,

(ii) $R_{1} \leq R_{2}$ (i.e., $R_{1}(x, y) \leq R_{2}(x, y), x, y \in \bar{S}$ with $x \sim y$ ).

Then

(i) $J_{1}(x, y)=J_{2}(x, y), x, y \in \bar{S}$,

(ii) $R_{1}(x, y)=R_{2}(x, y)$ whenever $J_{1}(x, y) \neq 0$ or $J_{2}(x, y) \neq 0$.

Proof. (i) By the Energy Conservation,

$$
\begin{aligned}
E_{R_{1}}\left(J_{1}\right) & =\sum_{z \in \partial S} \sigma(z) J_{1}(z) \\
& =\sum_{z \in \partial S} \sigma(z) J_{2}(z) \\
& =E_{R_{2}}\left(J_{2}\right) .
\end{aligned}
$$


Then it follows that

$$
\begin{aligned}
E_{R_{1}}\left(J_{1}\right) & =E_{R_{2}}\left(J_{2}\right) \\
& =\frac{1}{2} \sum_{x, y \in \bar{S}}\left[J_{2}(x, y)\right]^{2} R_{2}(x, y) \\
& \geq \frac{1}{2} \sum_{x, y \in \bar{S}}\left[J_{2}(x, y)\right]^{2} R_{1}(x, y) \\
& =E_{R_{1}}\left(J_{2}\right),
\end{aligned}
$$

which implies that $J_{2}$ is also a minimizer of $E_{R_{1}}$. Then by the Dirichlet principle, we have $J_{1}=J_{2}$.

(ii) Since $J_{1}=J_{2}$ by (i), it follows from (3.4) that $E_{R_{1}}\left(J_{1}\right)=E_{R_{2}}\left(J_{1}\right)$, which means that

$$
\sum_{x, y \in \bar{S}}\left[J_{1}(x, y)\right]^{2}\left[R_{2}(x, y)-R_{1}(x, y)\right]=0 .
$$

Therefore, $R_{1}(x, y)=R_{2}(x, y)$ whenever $J_{1}(x, y) \neq 0$. We are done.

Remark 3.4. A natural application of the above result is to check the connectivity of electrical circuits. Let $G=(\bar{S}, R ; \partial S)$ be the designed resistor network and $\widetilde{G}=(\bar{S}, \widetilde{R} ; \partial S)$ be a manufactured one. If $\widetilde{G}$ is damaged so that there occurs a disconnected resistor on its interior, then $R(x, y)<\infty=\widetilde{R}(x, y)$ for some $x, y \in S$ and $R \leq \widetilde{R}$. Hence the above result can be applied to check if there is a disconnected resistors or not.

Acknowledgement. The author would like to express his sincere gratitude to the referees for their valuable comments and suggestions.

\section{References}

[1] G. Alessandrini, Remark on a paper by H. Bellout and A. Friedman, Boll. Un. Mat. Ital. A (7) 3 (1989), no. 2, 243-249.

[2] H. Bellout and A. Friedman, Identification problems in potential theory, Arch. Rational Mech. Anal. 101 (1988), no. 2, 143-160.

[3] N. L. Biggs, Algebraic Graph Theory, Second edition. Cambridge Mathematical Library. Cambridge University Press, Cambridge, 1993.

[4] _ Potential theory on distance-regular graphs, Combin. Probab. Comput. 2 (1993), no. 3, 243-255.

[5] _ Algebraic potential theory on graphs, Bull. London Math. Soc. 29 (1997), no. $6,641-682$.

[6] J. A. Bondy and R. L. Hemminger, Graph reconstruction-a survey, J. Graph Theory 1 (1977), no. 3, 227-268.

[7] A. P. Calderón, On an inverse boundary value problem, Seminar on Numerical Analysis and its Applications to Continuum Physics (Rio de Janeiro, 1980), pp. 65-73, Soc. Brasil. Mat., Rio de Janeiro, 1980.

[8] F. R. K. Chung, Spectral graph theory, CBMS Regional Conference Series in Mathematics, 92. Published for the Conference Board of the Mathematical Sciences, Washington, DC; by the American Mathematical Society, Providence, RI, 1997. 
[9] F. R. K. Chung, M. Garrett, R. Graham, and D. Shallcross, Distance realization problems with applications to internet tomography, J. Comput. System Sci. 63 (2001), 432448.

[10] F. R. K. Chung and R. P. Langlands, A combinatorial Laplacian with vertex weights, J. Combin. Theory Ser. A 75 (1996), no. 2, 316-327.

[11] F. R. K. Chung and K. Oden, Weighted graph Laplacians and isoperimetric inequalities, Pacific J. Math. 192 (2000), no. 2, 257-273.

[12] F. R. K. Chung and S.-T. Yau, Discrete Green's functions, J. Combin. Theory Ser. A 91 (2000), no. 1-2, 191-214.

[13] D. M. Cvetkovic, M. Doob, I. Gutman, and A. Torgasev, Recent Results in The Theory of Graph Spectra, Annals of Discrete Mathematics, 36. North-Holland Publishing Co., Amsterdam, 1988.

[14] D. M. Cvetkovic, M. Doob, and H. Sachs, Spectra of Graphs, Theory and application. Pure and Applied Mathematics, 87. Academic Press, Inc. [Harcourt Brace Jovanovich, Publishers], New York-London, 1980.

[15] P. G. Doyle and J. L. Snell, Random Walks and Electric Networks, Carus Mathematical Monographs, 22. Mathematical Association of America, Washington, DC, 1984.

[16] S. L. Hakimi and S. S. Yau, Distance matrix of a graph and its realizability, Quart. Appl. Math. 22 (1965), 305-317.

[17] V. Isakov, Inverse Problems for Partial Differential Equations, Applied Mathematical Sciences, 127. Springer-Verlag, New York, 1998.

[18] V. Isakov and J. Powell, On the inverse conductivity problem with one measurement, Inverse Problems 6 (1990), no. 2, 311-318.

[19] J. Sylvester and G. Uhlmann, A global uniqueness theorem for an inverse boundary value problem, Ann. of Math. (2) 125 (1987), no. 1, 153-169.

Department of Mathematics and the Program of Integrated Biotechnology SOGANG UNIVERSITY SEOUl 121-742, Korea

E-mail address: sychung@sogang.ac.kr 